\title{
Problematisches Spielverhalten - Prävalenz und Verteilung
}

Fragestellung: Haben Patienten mit bipolarer Störung ein erhöhtes Risiko für exzessives und problematisches Spielverhalten und können hinsichtlich der Prävalenz Unterschiede zwischen den Subtypen der bipolaren Störung festgestellt werden?

Hintergrund: Nordamerikanischen Studien zur Folge ist die Rate von problematischem Spielverhalten bei Patienten mit bipolarer Störung im Vergleich zur Normalbevölkerung erhöht. Bislang ist wenig über die entsprechenden Prävalenzen bei den verschiedenen Subtypen der bipolaren Störung bekannt. Als problematisches Spielverhalten gilt exzessives Spielen, das zu individuellen, familiären oder sozialen Beeinträchtigungen führt. In den Jahren 2007 bis 2010 konnte in Großbritannien und Nordirland ein leichter Anstieg (von 0,5\% auf 0,7\%) verzeichnet werden. Es bestand daher die Notwendigkeit die Faktoren, die ein problematisches Spielverhalten begünstigen, genauer $\mathrm{zu}$ untersuchen. Affektive Störungen sind bekanntermaßen mit einer erhöhten Prävalenz für problematisches Spielverhalten verbunden. Bislang ist jedoch in Bezug auf die Prävalenz keine Differenzierung zwischen

Jones L, Metcalf A, GordonSmith K et al. Gambling problems in bipolar disorder in the UK: prevalence and distribution Br J Psychiatry 2015; 207: $328-33$ den unterschiedlichen Entitäten beziehungsweise den Subtypen der bipolaren Störung vorgenommen worden. Die vorliegende Studie ist die erste, die die Prävalenz problematischen Spielverhaltens bei Patienten mit bipolarer Störung vom Typ 1 im Vergleich zum Typ 2 untersucht.

Patienten und Methodik: Die Rekrutierung der Patienten erfolgte aus dem Bipolar Disorder Research Network (BDRN, britisches Netzwerk und Forschungsprogramm zur Untersuchung genetischer und nicht genetischer Aspekte affektiver Störungen). 635 Patienten erfüllten die Einschlusskriterien. Zur Beurteilung des Schweregrades problematischen Spielverhaltens wurde der Problem Gambling Severity Index (PGSI) herangezogen.

Ergebnisse: Mittelschwere bis schwere Beeinträchtigungen infolge problematischen Spielverhaltens traten bei Patienten mit bipolarer Störung insgesamt bis zu vierfach häufiger auf als in der Allgemeinbevölkerung. Schwere Beeinträchtigungen waren im Wesentlichen assoziiert mit bipolarer Störung II (Odds Ratio $[\mathrm{OR}]=1,74, \mathrm{p}=0,036)$, Suizidgedanken oder -versuchen in der Anamnese $(\mathrm{OR}=3,44, \mathrm{p}=0,02)$ und Rapid Cycling $(\mathrm{OR}=2,63$, $\mathrm{p}=0,008)$.

Schlussfolgerungen: Schätzungsweise hat einer von zehn Patienten mit bipolarer Störung ein erhöhtes Risiko ein problematisches Spielverhalten zu entwickeln. Das ist assoziiert mit Suizidalität und Rapid Cycling. Die erhöhte Prävalenz insbesondere bei bipolarer Störung II unterstreicht, dass auch mildere Formen affektiver Störungen die Entwicklung von Verhaltensauffälligkeiten begünstigen können.

\section{- Kommentar von Julia Becker-Sadzio, Tübingen}

\section{Spielverhalten bei bipolarer Störung klinisch berücksichtigen}

Die vorliegende Studie befasst sich als erste mit der Prävalenz von problematischem Spielverhalten bei Patienten mit bipolarer Störung und differenziert hier zwischen den verschiedenen Subtypen der Erkrankung. Die Zahl der Probanden ist groß und repräsentativ. Die Ergebnisse müssen dennoch vor dem Hintergrund verschiedener potenzieller Unschärfen kritisch hinterfragt werden. Bei dem verwendeten Fragebogen handelt es sich beispielsweise um einen Selbstbeurteilungsfragebogen, sodass die vermutete soziale Erwünschtheit der Antworten und eine mögliche Erinnerungsverzerrung (recall bias) die Ergebnisse beeinflusst haben könnten. Weiterhin nahmen lediglich $23 \%$ der eingeladenen Probanden an der Befragung teil. Ob das zu einer Über- oder Unterschätzung der Prävalenz beigetragen hat, ist allerdings schwer zu beurteilen. Eine Aussage hinsichtlich der Kausalität kann nicht getroffen werden, sodass ergänzende Längsschnittstudien notwendig sind, um den Zusammenhang zwischen affektiven Störungen und problematischem Spielverhalten genauer zu untersu- chen. Insgesamt sind die vorliegenden Ergebnisse dahingehend von klinischem Interesse, als dass sie dazu anregen können, gerade bei Patienten mit bipolarer Störung ein problematisches Spielverhalten insbesondere durch exzessives Nutzen neuer Medien quantitativ und qualitativ regelhaft in der Anamneseerhebung zu explorieren.

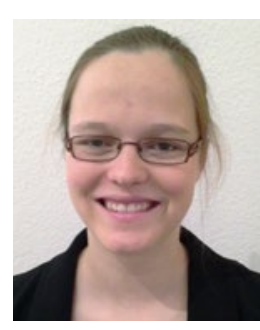

Julia Becker-Sadzio, Tübingen

Klinik für Psychiatrie und Psychotherapie Universitätsklinikum Tübingen

E-Mail: Julia.Sadzio@med.uni-tuebingen.de 